\title{
Automated Complex of Motion Control Based on Vibrotactile Feedback for Rehabilitiation
}

DOI: $10.17691 / \mathrm{stm} 2017.9 .4 .04$

Received September 7, 2017

D. V. Kutyina, Engineer, Laboratory of Neuroengineering, Center for Translational Technologies';

D.G. Nakonechny, MD, PhD, Researcher, Head of Hand and Foot Surgery Department2;

A. Motailo, Assistant, Laboratory for Brain-Machine Interface Development,

Center for Translational Technologies';

Ya.1. Pigareva, Junior Researcher, Laboratory of Neuroengineering, Center for Translational Technologies';

A.N. Kiseleva, Orthopedic Surgeon, Hand and Foot Surgery Department2;

V.B. Kazantsev, DSC, Vice-Rector for Research'; Head of the Department of Neurotechnologies,

Institute of Biology and Biomedicine; Head of the Laboratory for the Development of Intellectual

Biomechatronic Technologies, Center for Translational Technologies';

S.Yu. Gordleeva, PhD, Researcher, Laboratory for Brain-Machine Interface Development,

Center for Translational Technologies';

A.Ya. Kaplan, DSc, Head of the Laboratory of Neurophysiology and Neuro-Computer Interfaces ${ }^{3}$;

Head of the Laboratory for Brain-Machine Interface Development', Center for Translational Technologies';

A.S. Pimashkin, PhD, Head of the Laboratory of Neuroengineering, Center for Translational Technologies ${ }^{1}$

'Lobachevsky State University of Nizhni Novgorod, 23 Prospekt Gagarina, Nizhny Novgorod, 603950,

Russian Federation;

${ }^{2}$ Russian Scientific Research Institute of Traumatology and Orthopedics named after R.R. Vreden,

8 Academik Baikov St., Saint Petersburg, 195427, Russian Federation;

${ }^{3}$ Lomonosov Moscow State University, 1 Leninskiye Gory, Moscow, 119991, Russian Federation

This report presents a technique for using vibrotactile feedback for rehabilitation of patients after an operation on the hand with a suture on the tendon. The task is to limit the arm muscle tension using vibrotactile feedback, which aims to prevent exceeding the preset force threshold and thus protect the stitched tendons from rupture in the post-operation period.

The study goals were to develop an automated complex able to record muscle activity (myography) and induce vibrotactile signals and develop an algorithm able to inform the patient (via tactile sensation) that the muscle tension is exceeded. These developments were aimed at preventing possible tendon rupture and maintaining smooth recovery after surgery.

Materials and Methods. A wireless system consisting of a single-channel myograph combined with a tactile pulse generator (vibration actuator) was used. The system was placed on the forearm and was controlled from a remote computer in both manual and automatic modes using a software package developed in the MATLAB environment. The real-time analysis of the myographic signal allowed us to determine the force of muscle contraction. When the preset threshold $(20 \%$ of the maximum) is exceeded, the system triggers a short burst (200 ms duration) of vibration pulses. This vibration stimulus informs the subject about the exceeding or redundant muscle tension, after which he/she stops further exercise.

Results. Here we show that the vibrotactile feedback signal lasting hundreds of milliseconds is well perceived by the subject and allows him/her to respond so not to exceed the preset muscle force threshold. This biological feedback is viewed as physiologically favorable for patients because it can automatically inform them about excessive muscle contractions that are undesirable in the rehabilitation period. In the long term, this feedback mechanism may help forming normal patterns in patients.

Key words: vibrotactile feedback; neurorehabilitation; surface electromyography; tendon restoration.

The use of biofeedback (BFB) is a relatively recent trend in rehabilitation medicine; it has been often employed for rehabilitation of patients after injuries or disorders of the musculoskeletal and nervous systems. These categories of patients are in need of additional monitoring and control of their muscle functions.

It has been shown that signals coming from the human tactile system are perceived by the body more readily than other types of physiological feedback, and are able to substitute natural proprioception in regulating muscle contractions. In this case, tactile, vibrotactile or electrotactile signals as well as their combinations can be used [1, 2]. Such signals are most commonly used for training and rehabilitation of patients with upper

For contacts: Alexey S. Pimashkin, e-mail: pimashkin@neuro.nnov.ru 
limb prostheses because in these cases a feedback is necessary not only for training but also for normal functioning of the artificial limb [3, 4].

The BFB approach is relevant for the transplanted limb, which, in terms of biomechanics, is similar to a bionic prosthesis except for some biologically determined features [5]. There are more clinical situations, in which the use of BFB is beneficial (a procedure supplemental to physiotherapy or in preparation for forthcoming treatment) [6]. For example, after arm tendon surgery, the use of BFB may be helpful for successful healing and rehabilitation. Tendon suture is performed in tendon injuries and their consequences, as well as transpositions of tendons after nerve injuries at various levels [7-10]. In these cases, it is necessary, on the one hand, to provide sufficient mobility to the tendons and prevent adhesions, and, to protect the suture zone from excessive tension to avoid its failure. For this purpose, rehabilitation protocols of postoperative management are designed to provide the newly stitched tendons with maximal yet safe exercise [11-15]. However, surgeons and physiotherapists can decide on the degree of mobility based only on the data from mechanical studies in vitro or retrospective data of others on the effectiveness of rehabilitation $[16,17]$.

Muscle tension is mainly controlled by passive measures like limitation of muscular activity by excluding certain movements, or imposing immobilization, or using passive elastic bands [18]. These measures are prescribed and supervised by a rehabilitation specialist. However, in the absence of such an expert (e.g., at home), the patient cannot fully control his/her muscle tone, which increases the likelihood of suture rupture.

Continuous monitoring of muscular tension is needed to create the optimal conditions for rehabilitation of patients after tendon injuries, or compensate for the function of paralyzed muscles in tendon-muscle transfers. This would allow to monitor and control muscle tension and force applied to the tendon junction, help create new motor stereotypes, reduce the probability of suture failure, and retrain the transposed muscles to function.

The BFB devices provide the patient with a permanent connection to his/her muscles via visual or audible feedback systems [19]. Before introducing the systems with tactile feedback into the area of postsurgery tendon rehabilitation, the effectiveness of tactile BFB was demonstrated in the brain-machine interface setting based on EEG signals [20]. The vibration stimulus used for the feedback formation is very close to physiological proprioception. This approach seems quite promising for the development of automated methods of personalized rehabilitation, because it provides tools to monitor/control several areas of patient's body, simultaneously. Moreover, when using a vibrotactile BFB system the patient does not need any other audiovisual sensory channel to ensure its continuous functioning at home. The development of such brain-machine neuro-interfaces has recently become one of the most promising areas in neurological rehabilitation. Research into this field is based on novel technologies for recording neurophysiological signals and a better understanding of neuroplasticity and information processing in the brain and nervous system [21].

\section{Materials and Methods}

Determination of vibration characteristics. To assess the perception of vibration stimuli and to optimize the vibration parameters we have developed a device based on a microcontroller (Atmel ATMega328P; Atmel, USA) that guided the vibrator (Figure 1 (a)). In the experiment, a LRA motor with a diameter of $10 \mathrm{~mm}$ was used as a vibration actuator. The motor was held with a retainer on the medial side of the subject's forearm. During the experiment, the subject was asked to put his/ her arm on the table and relax the arm muscles. The intensities of the vibration stimuli were controlled by means of pulse-width modulation. As the pulse duration increased (the vibrator was active for longer time) the generated stimuli were perceived as more intense (Figure 1 (b)). The microcontroller thus set the operating mode of the vibration actuator.

To assess the perception of vibrations, we proposed a perception-evaluating scale with four grades of sensations: imperceptible, weakly perceptible, well perceptible (optimal) and unpleasant. In the developed software for guiding the microcontroller, the duration of the vibration pulses increased incrementally during the experiment by steps of $0.2 \mathrm{~ms}$ from the minimum to the maximum (2 ms). The pulse frequency was set to $500 \mathrm{~Hz}$. With each change in the pulse duration, the subjects were asked to evaluate their sensations according to the proposed perception scale.

In total, 7 subjects took part in the experiment. The study was carried out in accordance with the Helsinki Declaration, adopted in June 1964 (Helsinki, Finland) and revised in October 2000 (Edinburgh, Scotland), and approved by the Ethics Committee of the Lobachevsky State University of Nizhni Novgorod. An informed consent was obtained from each patient. The experimentation on vibration perceptions lasted for 3 days. Three recordings were made for each day with an interval of $1 \mathrm{~h}$. Statistical analysis of the experimental data was performed in MATLAB and Statistica 6.0.

Biological feedback with vibrotactile stimulation. To test the feasibility of BFB for muscle contraction, we developed a hardware-software complex, which consisted of an electromyographic recording device and a vibration actuator (Figure 2). The functional algorithm of the complex included a continuous recording/ analysis of muscle activity and a vibration control. If a preset threshold of muscle contraction is exceeded, the vibrotactile signal is turned on.

In the experiment, the subject was asked to perform a series of monotonous exercises, such as periodically squeezing and relaxing a hand-gripper. We used a $260 \mathrm{~A}$ hand-gripper (Pro-Supra, China) capable of adjusting the 

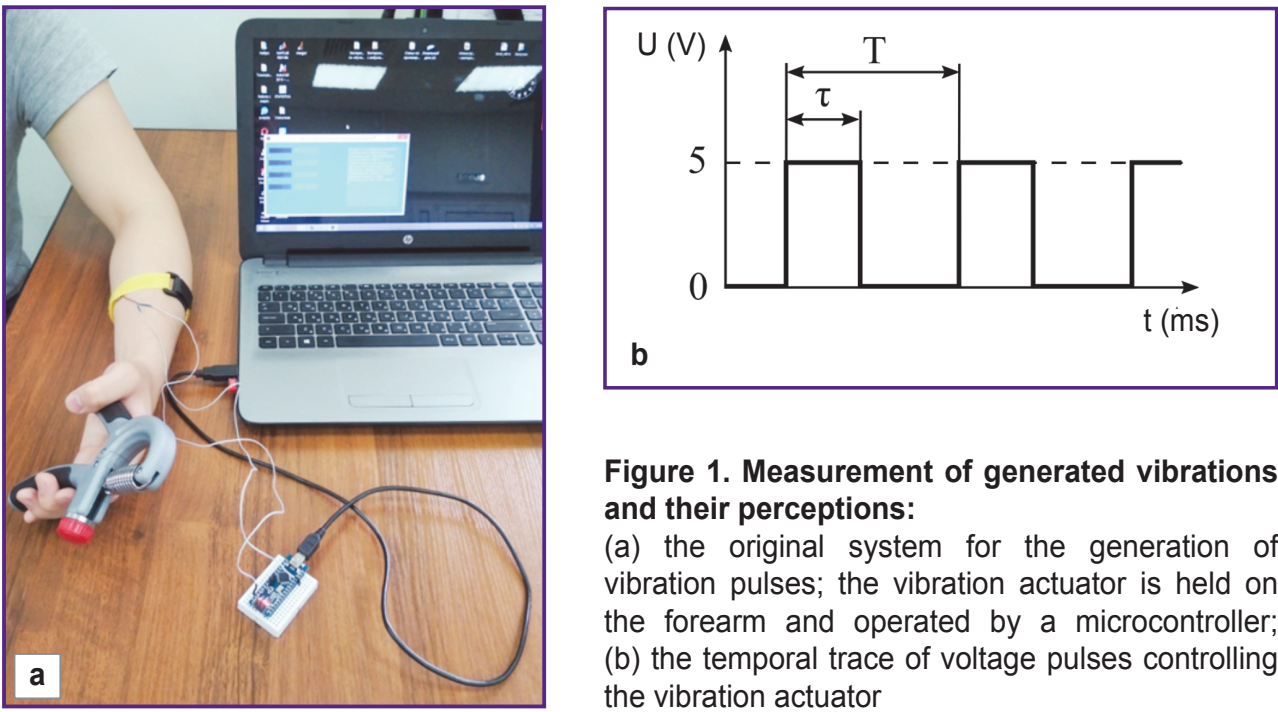

Figure 1. Measurement of generated vibrations and their perceptions:

(a) the original system for the generation of vibration pulses; the vibration actuator is held on the forearm and operated by a microcontroller; (b) the temporal trace of voltage pulses controlling the vibration actuator
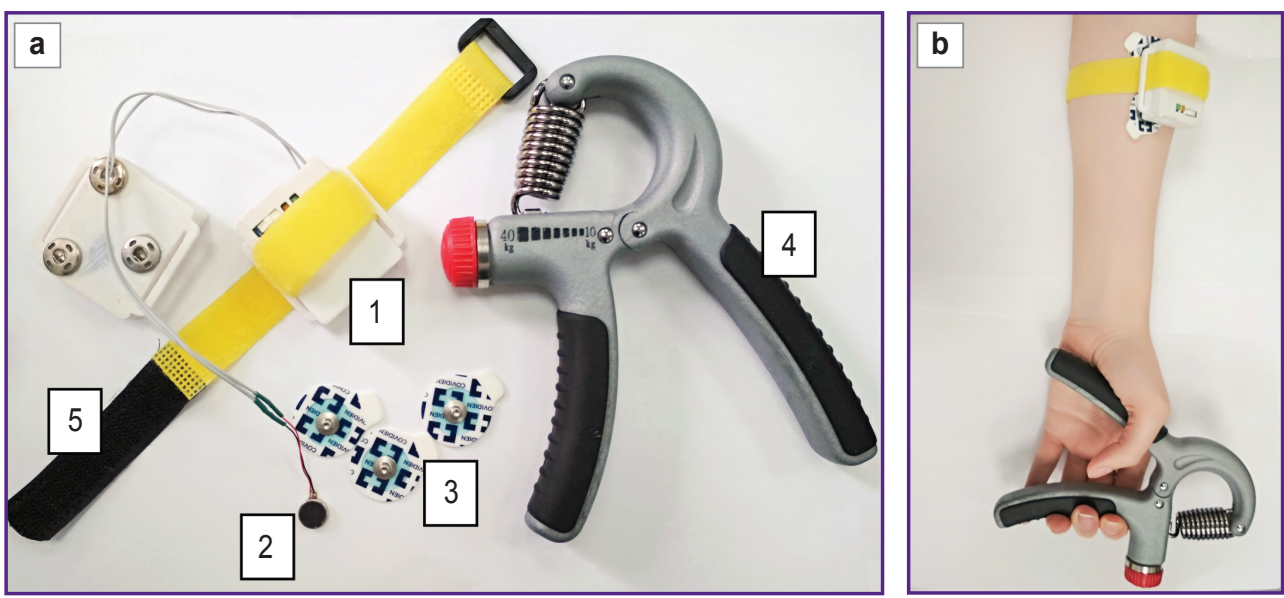

Figure 2. The system for muscle contraction estimation using surface electromyography: (a) the biopotentials were measured using medical electrodes (3) and a single-channel biopotential amplifier (1) in the bipolar mode; to induce muscular contraction, a hand-gripper was used (4); the vibration stimuli were sent to a vibrating actuator (2) held with an elastic tape (5) on the medial side of the subject's forearm; (b) the system at work

load within a range from 10 to $40 \mathrm{~kg}$. For the experiment, a minimum load of $10 \mathrm{~kg}$ was selected.

To record the bioelectric muscle activity we developed an amplifier of electromyographic signals. The amplifier recorded the signals recorded by medical electrodes (H124SG Kendall ${ }^{\mathrm{TM}}$; USA) with a surface area of $30 \times 24 \mathrm{~mm}$ and an $\mathrm{AgCl}$-coating. The amplifier was placed on the pre-cleaned skin of the forearm. The distance between the electrodes was $2 \mathrm{~cm}$ as determined by the design of the amplifier (Figure $2(b)$ ).

Signals of muscle activity were processed in real time with a computer using custom-made software developed in the MATLAB environment. The main characteristic of the muscle contraction force was estimated as RMS (root-mean-square) of the signal values in the time window of $200 \mathrm{~ms}$. Also, the threshold of the contraction force was defined as percent of the maximal force.
According to the proposed feedback mode, when the RMS values exceeded the preset threshold, the vibration actuator triggered on.

Experimental protocol. Before beginning the experiment, we normalized the muscle activity. The subject maximally squeezed the hand-gripper, kept this tension level for a few seconds and then relaxed his grip. In this exercise, the system recorded the maximal and minimal RMS values, which were then taken for 0 and $100 \%$ of the muscle tension, respectively.

The experiment was performed both without the vibration feedback, and with it. In the first case, the subject periodically squeezed and relaxed the handgripper, guided by his/her own sensations, to a level of $20 \%$ of the maximal muscle tension. With the vibration feedback incorporated into the system, when the preset level was exceeded, vibration was activated. 
The vibration perceived by the subject signaled that the preset threshold of muscle tension was exceeded and that the subject was required to reduce muscle tension in exercise. It is known that a long-time exercise is followed by a decrease in the working capacity of muscles; therefore we limited the duration of the experiment by $1 \mathrm{~min}$. During that period, the subject's muscles did not reach the levels of fatigue; accordingly, the force of muscle contractions and the level of bioelectrical activity did not change.

During the experiment, electromyographic signals were recorded for further analysis. To test the quality of the feedback system, we counted the number of data points when the real RMS values exceeded the preset threshold. The number of such supra-threshold values was normalized to the total number of recorded RMS values and was defined as a "percentage of errors".

\section{Results and Discussion}

Threshold of optimal perception under vibrostimulation. To determine the optimal parameters of vibration feedback, the perception of vibrations of different intensities was monitored. We proposed a number of requirements for vibration pulses: they should not cause unpleasant sensations, and they should be sensed by the subject both when relaxed and during exercise (periodic muscle contractions). The main characteristic that determines the intensity of vibrations is the pulse duration (see "Materials and Methods"). To assess the subject's sensitivity to vibration, four levels of subjective perception were introduced: imperceptible, perceptible only with subject concentration on vibration, perceptible during exercise and unpleasant (irritating). As a result of the experiment, the cut-off values indicating the boundaries between these levels of perception were obtained.

The vibrations perceived as "mild" on average were greater than $0.5 \mathrm{~ms}$ (Figure 3, blue graph).

Then the vibration intensity was enhanced by increasing the pulse durations. At that time, the sensations were recorded as reported by the subjects who focused his/her attention on vibration. At the next step, the subject was asked to squeeze the gripper and report the sensations. At rest, the vibrations were perceived through the entire range of pulse durations. At exercise though, the vibration was clearly perceived starting (on average) above $0.8 \mathrm{~ms}$ (Figure 3, green graph). At high intensities of vibration stimuli, the subjects reported unpleasant or irritable sensations. This (unpleasant) level of intensity was defined as the maximum (Figure 3, red graph) and varied from 0.8 to $2 \mathrm{~ms}$ (mean value was equal to $1.5 \mathrm{~ms}$ ). Some subjects perceived vibrations generated by the vibration actuator, as comfortable even with a maximal pulse duration of $2 \mathrm{~ms}$. The experiments were carried out for three days.
Each day, three tests were performed at 1-hour intervals. There were no significant changes of boundaries between the vibration perception intervals from day to day or from hour to hour (ANOVA test, $p>0.05$ ).

As a result, an intensity interval (from 0.8 to $1.6 \mathrm{~ms}$ ) was found in which the vibrations were well perceived but caused no irritation. In the subsequent feedback experiments, the middle-range pulse $(1.2 \mathrm{~ms})$ was used.

Efficiency of the vibrotactile feedback. At the next stage, we studied the closed-loop system based on the vibrostimulation feedback. The main task was to create a system able to limit the muscle tension when the force of muscle contractions exceeded the threshold of $20 \%$ of the maximum. In this system, the vibration acted as a signal informing the subject that his/her muscle tension has exceeded the safe threshold; the subject was then supposed to reduce his/her muscle work. The experiment included squeezing-relaxing the gripper and simultaneously recording the electromyographic activity of subject's forearm flexor muscles. To confirm the efficacy of the proposed feedback, two recordings were made: without and with the feedback. In the first run, the subject was asked to not exceed the threshold of muscle tension, as per his/her own judgement. Figure 4 (a) (on the left) shows the trace of RMS values; the dotted line indicates the previously estimated threshold. It can be seen that with each contraction, the RMS values exceed the preset threshold. Then, the experiment was re-run with the vibration feedback turned on. Here, the optimal pulse duration of $1.2 \mathrm{~ms}$ (found earlier, see Figure 2) was used. At the time the tension threshold was exceeded, the vibrostimulation got activated and continued until the RMS values dropped below the threshold level. In Figure 4 (a) (on the right), the trace of this experiment is

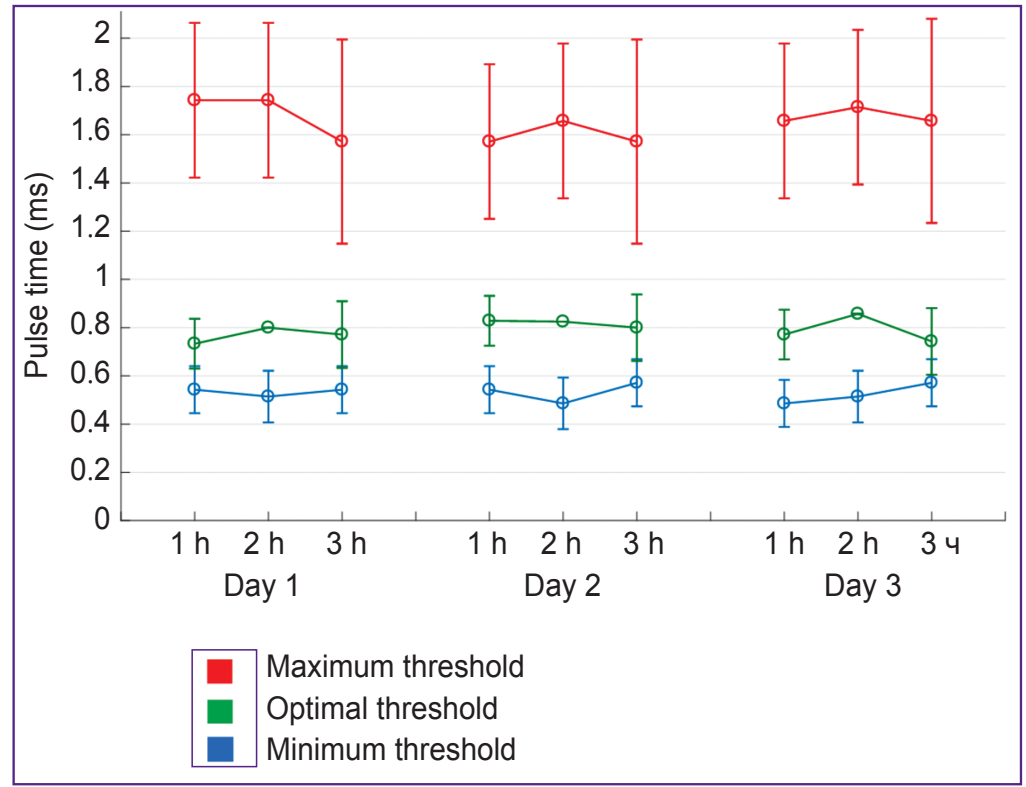

Figure 3. Changes in threshold levels of vibrostimulation perception during 3 days period 


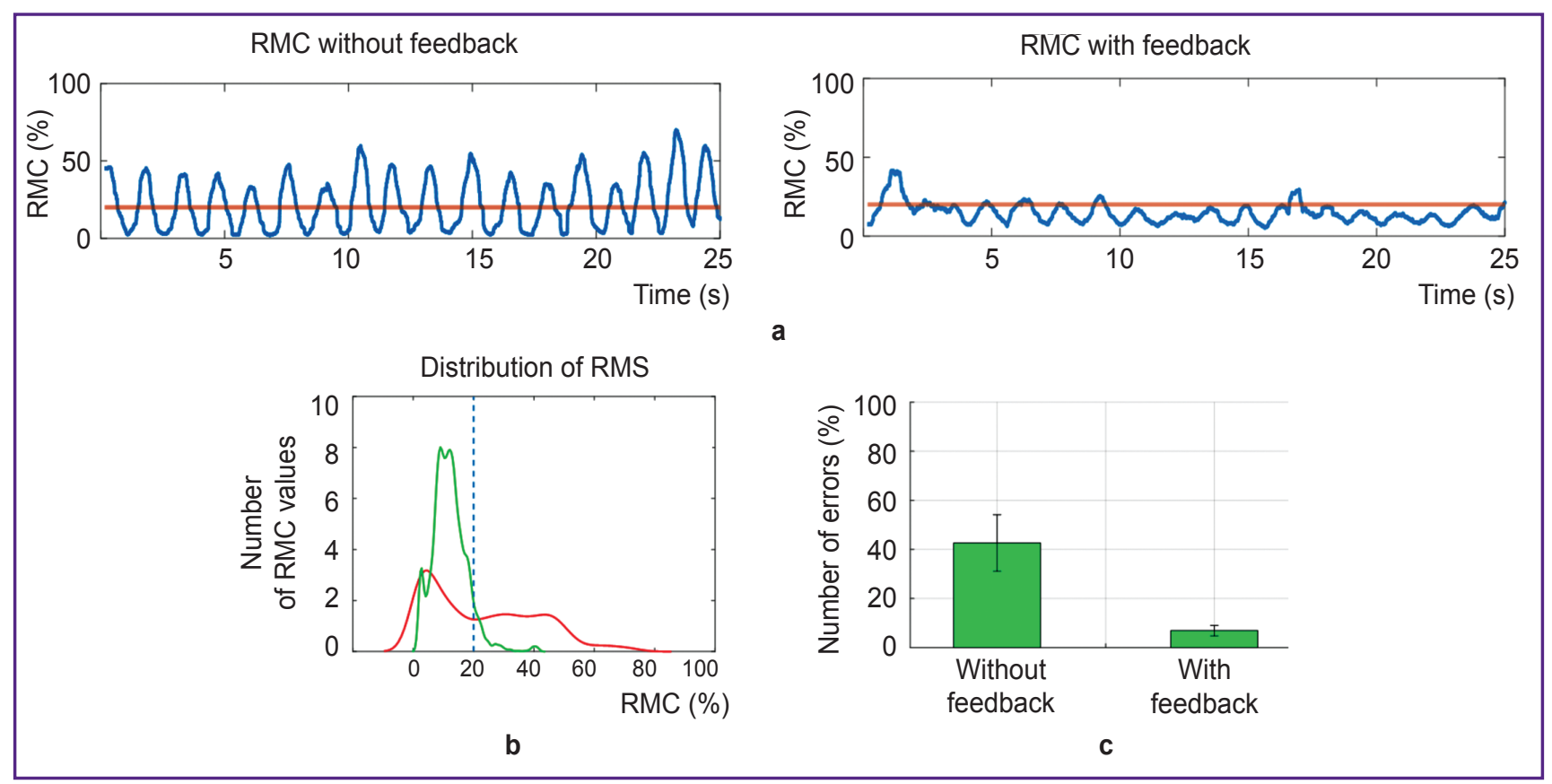

Figure 4. Efficiency of vibrotactile feedback for the control of muscle contraction:

(a) the traces of RMS recordings without and with the vibrotactile feedback, the horizontal red lines indicate the preset thresholds of $20 \%$; (b) distribution of RMS values recorded without (red line) and with (green line) the feedback, the dotted vertical line shows the threshold of $20 \%$; (c) percentage of errors made without and with the feedback component

presented. It shows that after the initial supra-threshold (erroneous) contractions, the subsequent muscle activity was almost entirely below the threshold value.

To illustrate the differences in muscle activity in the absence or presence of the feedback, we present the distribution of the RMS values in these two recordings (Figure 4 (b)). The results indicate that most of RMS values controlled by the feedback are below the threshold (dotted vertical line); without feedback the distribution curve looks different. To quantify the feedback efficiency, we counted the number of (erroneous) values that exceeded the threshold level. The percentage of errors $(7 \pm 2 \%, n=3)$ clearly reflected the effectiveness of the feedback system in limiting the muscle contraction (Figure 4 (c)). Contrary to that in the case without feedback the percentage of errors averaged at $42 \pm 12 \%$; the difference between the two cases was statistically significant (t-test, $p<0.01)$. This result led us to conclude that the system with vibrotactile feedback was significantly more effective in controlling muscle tension than the consciousness-driven control without any devices and based on subjective sensation.

Conclusion. Local vibrotactile stimulation using a miniature vibration actuator can be used as a biological feedback in medical research. The perception of vibrational pulses of various intensities remains fairly constant for several hours or even days, which validates using this type of information signal in human subjects. The vibrotactile feedback estimated from an electromyographic signal is perceived by a subject within hundreds of milliseconds and allows him/her to not exceed the preset muscle contraction threshold. The system is able to autonomously inform a person of undesirable and dangerous muscle contractions that may improve rehabilitation process. This biological feedback is physiologically reliable for human perception and can provide normal motor patterns formation.

Financial Support. The study was supported by the Russian Science Foundation (grant \#15-19-20053).

Conflict of Interest is not declared.

\section{References}

1. Maaswinkel E., Veeger H.E.J., Dieen J.H. Interactions of touch feedback with muscle vibration and galvanic vestibular stimulation in the control of trunk posture. Gait Posture 2014; 39(2): 745-749, https://doi.org/10.1016/j. gaitpost.2013.10.011.

2. Štrbac M., Belić M., Isaković M., Kojić V., Bijelić G., Popović I., Radotić M., Došen S., Marković M., Farina D., Keller T. Integrated and flexible multichannel interface for electrotactile stimulation. J Neural Eng 2016; 13: 046014, https://doi.org/10.1088/1741-2560/13/4/046014.

3. Li K., Fang Y., Zhou Y., Liu H. Non-invasive stimulationbased tactile sensation for upper-extremity prosthesis: a review. IEEE Sensors Journal 2017; 17(9): 2625-2635, https:// doi.org/10.1109/jsen.2017.2674965.

4. Svensson P., Wijk U., Björkman A., Antfolk C. A review of invasive and non-invasive sensory feedback in upper limb 
prostheses. Expert Rev Med Devices 2017; 14(6): 439-447, https://doi.org/10.1080/17434440.2017.1332989.

5. Kurzynski M., Jaskolska A., Marusiak J., Wolczowski A., Bierut P., Szumowski L., Witkowski J., Kisiel-Sajewicz K. Computer-aided training sensorimotor cortex functions in humans before the upper limb transplantation using virtual reality and sensory feedback. Comput Biol Med 2017; 87: 311-321, https://doi.org/10.1016/j. compbiomed.2017.06.010.

6. Liyanagamage S.A., Bertucco M., Bhanpuri N.H., Sanger T.D. Scaled vibratory feedback can bias muscle use in children with dystonia during a redundant, 1-dimensional myocontrol task. J Child Neurol 2017; 32(2): 161-169, https:// doi.org/10.1177/0883073816671830.

7. Giesen T., Calcagni M., Elliot D. Primary flexor tendon repair with early active motion. Hand Clinics 2017; 33(3): 465472, https://doi.org/10.1016/j.hcl.2017.03.001.

8. Makarewich C.A., Hutchinson D.T. Tendon transfers for combined peripheral nerve injuries. Hand Clinics 2016; 32(3): 377-338, https://doi.org/10.1016/j.hcl.2016.03.008.

9. Sammer D.M., Chung K.C. Tendon transfers: part II. transfers for ulnar nerve palsy and median nerve palsy. Plast Reconstr Surg 2009; 124(3): 212e-221e, https://doi. org/10.1097/prs.0b013e3181b037c7.

10. Dunn J.A., Sinnott K.A., Rothwell A.G., Mohammed K.D., Simcock J.W. Tendon transfer surgery for people with tetraplegia: an overview Arch Phys Med Rehabil 2016; 97(6): S75-S80, https://doi.org/10.1016/j.apmr.2016.01.034.

11. Starr H.M., Snoddy M., Hammond K.E., Seiler J.G. Flexor tendon repair rehabilitation protocols: a systematic review. J Hand Surg Am 2013; 38(9): 1712-1717.e14, https:// doi.org/10.1016/j.jhsa.2013.06.025.

12. Higgins A., Lalonde D.H. Flexor tendon repair postoperative rehabilitation. Plast Reconstr Surg Glob Open 2016; 4(11): e1134, https://doi.org/10.1097/gox. 0000000000001134.
13. Wangdell J., Bunketorp-Käll L., Koch-Borner S., Fridén J. Early active rehabilitation after grip reconstructive surgery in tetraplegia. Arch Phys Med Rehabil 2016; 97(6): S117-S125, https://doi.org/10.1016/j.apmr.2015.09.025.

14. Von der Heyde R., Novak C. Rehabilitation of the upper extremity following nerve and tendon reconstruction: when and how. Semin Plast Surg 2015; 29(01): 073-080, https://doi. org/10.1055/s-0035-1544172.

15. Ahluwalia S.P., Pandey S., Sarad R., Boparai R.S. Flexor tendon repair rehabilitation protocols: a randomized prospective trial of Kleinert protocol compared with Duran protocol J Clin Orthop Trauma 2015; 6(1): 73, https://doi. org/10.1016/j.jcot.2015.01.053.

16. Tanaka T., Amadio P.C., Zhao C., Zobitz M.E., An K.-N. Flexor digitorum profundus tendon tension during finger manipulation. J Hand Ther 2005; 18(3): 330-338, https:// doi.org/10.1197/j.jht.2005.04.001.

17. Sultana S.S., MacDermid J.C., Grewal R., Rath S. The effectiveness of early mobilization after tendon transfers in the hand: a systematic review. J Hand Ther 2013; 26(1): 1-21, https://doi.org/10.1016/j.jht.2012.06.006.

18. Kleinert H.E., Kutz J.E., Atasoy E., Stormo A. Primary repair of flexor tendons. Orthop Clin North Am 1973; 4(4): 865-876.

19. Tripp B.L., Faust D., Jacobs P. Elbow joint position sense after neuromuscular training with handheld vibration. J Athl Train 2009; 44(6): 617-623, https://doi. org/10.4085/1062-6050-44.6.617.

20. Liburkina S.P., Vasilyev A.N., Yakovlev L.V., Gordleeva S.Y., Kaplan A.Y. Motor imagery based brain computer interface with vibrotactile interaction. Zhurnal vysshey nervnoy deyatel'nosti im. I.P. Pavlova 2017; 67(4): 414-429.

21. Kaplan A.Y. Neurophysiological foundations and practical realizations of the brain-machine interfaces in the technology in neurological rehabilitation. Hum Physiol 2016; 42(1): 103-110, https://doi.org/10.1134/s0362119716010102. 